\title{
Strong Matching Preclusion for Augmented Butterfly Networks
}

\author{
Jinyu Zou, Yan Sun, Chengfu Ye* \\ School of Computer Sciences, Qinghai Normal University, Xining, China \\ Email address: \\ jinyuZou@126.com (Jinyu Zou), sy0623@163.com (Yan Sun), yechengfu@yahoo.com (Chengfu Ye) \\ ${ }^{*}$ Corresponding author
}

To cite this article:

Jinyu Zou, Yan Sun, Chengfu Ye. Strong Matching Preclusion for Augmented Butterfly Networks. American Journal of Applied Mathematics. Vol. 7, No. 2, 2019, pp. 58-62. doi: 10.11648/j.ajam.20190702.13

Received: May 24, 2019; Accepted: June 27, 2019; Published: July 9, 2019

\begin{abstract}
The matching preclusion number of a graph is the minimum number of edges whose deletion results in a graph that has neither perfect matchings nor almost-perfect matchings. The strong matching preclusion number (or simply, SMP number) $\operatorname{smp}(G)$ of a graph $G$ is the minimum number of vertices and/or edges whose deletion results in a graph that has neither perfect matchings nor almost-perfect matchings. This is an extension of the matching preclusion problem and has been introduced by Park and Ihm. Butterfly Networks are interconnection networks which form the back bone of distributed memory parallel architecture. One of the current interests of researchers is Butterfly graphs, because they are studied as a topology of parallel machine architecture. Butterfly network has many weaknesses. It is non-Hamiltonian, not pancyclic and its toughness is less than one. But augmented butterfly network retains most of the favorable properties of the butterfly network. In this paper, we determine the strong matching preclusion number of the Augmented Butterfly networks.
\end{abstract}

keywords: Matching, Strong Matching Preclusion, Augmented Butterfly Networks

\section{Introduction}

Let $G$ be a connected graph, $V(G)$ and $E(G)$ are its vertex set and edge set respectively. A perfect matching is a set of edges such that every vertex is incident with exactly one edge in this set. An almost-perfect matching in a graph is a set of edges such that every vertex except one is incident with exactly one edge in this set, and the exceptional vertex is incident to none. In this paper, we say that such exceptional vertex isn't matched under the almost perfect matching.

All graphs considered in this paper are undirected, finite and simple. It is referred to the book [3] for graph theoretical notation and terminology not described here. Let $X$ be a set of vertices and edges. Let $G-X$ denote the subgraph of $G$ obtained by removing all the vertices of $X$ together with the edges incident with them from $G$ as well as removing all the edges of $X$ from $G$. If $X=v$ and $X=e$ where $v$ is a vertex and $e$ is an edge, simply wrote by $G-v$ and $G-e$ for $G-v$ and $G-e$, respectively. The degree of a vertex $v$ in a graph $\mathrm{G}$, denoted by $\operatorname{deg}_{G}(v)$, is the number of edges of $G$ incident with $v$. Let $\delta(G)$ be the minimum degree of the vertices of $\mathrm{G}$, respectively. A component of a graph is odd or even according to whether it has an odd or even number of vertices.

A set of edges $F$ is called a matching preclusion se $\mathrm{t}$ if $G-F$ has no perfect matching, and it is called an optimal matching preclusion set if $F$ is one with the smallest size. The matching preclusion number of an even graph $G$, denoted by $m p(G)$, is the minimum number of edges whose deletion leaves the resulting graph without a perfect matching. If $G$ has no perfect matchings, then $m p(G)=0$. The concept of matching preclusion was introduced by Brigham et al. [2], as a measure of robustness in the event of edge failure in interconnection networks. An interconnection network with a larger MP number may be considered as more robust in the event of link failures.

Proposition 1-1

Let $G$ be a graph with an even number of vertices. Then $m p(G) \leq \delta(G)$, where $\delta(G)$ is the minimum degree of $G$

The concept of strong matching preclusion was proposed by Park and Ihm [2]. A set $F$ of vertices and edges in a graph $G$ is called a strong matching preclusion set SMP set for short if $G-F$ has neither a perfect matching nor an almost perfect matching. The strong matching preclusion number of $G$, 
denoted by $\operatorname{smp}(G)$, is defined to be the minimum size of all possible SMP sets of $G$. Strong matching preclusion that additionally permits more destructive vertex faults in a graph is a more extensive form of the original matching preclusion that assumes only edge faults. According to the definition of $m p(G) \quad$ and $\operatorname{smp}(G)$, we have that $\operatorname{smp}(G) \leq m p(G) \leq \delta(G)$.

Park and Ihm [7] established the SMP number and all possible minimum strong matching preclusion sets for complete graphs, regular bipartite graphs, restricted HL-graphs, and recursive circulant graphs. Park and Ihm [8] also studied the problem of strong matching preclusion under the condition that no isolated vertex is created as a result of faults, and established the conditional strong matching preclusion number for the class of restricted hypercube-like graphs, which include most non bipartite hypercube-like networks found in the literature. SMP numbers of augmented cubes, arrangement graphs, alternating group graphs and split-star, pancake graphs, 2 -matching composition networks, $k$-ary $n$ cubes, $\mathrm{n}$-dimensional torus networks, k-composition networks are also investigated; see [1, 9-14].

In this paper, the strong matching preclusion number of the Augmented Butterfly networks is investigated.

\section{Augmented Butterfly Networks and Some Lemmas}

Butterfly network has many weakness. It is non-Hamiltonian, not pancyclic and its toughness is less than one. But augmented butterfly network retains most of the favorable properties of the butterfly network.

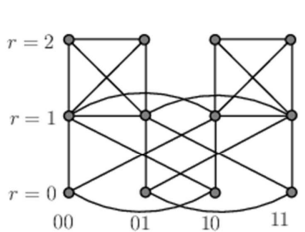

(1)

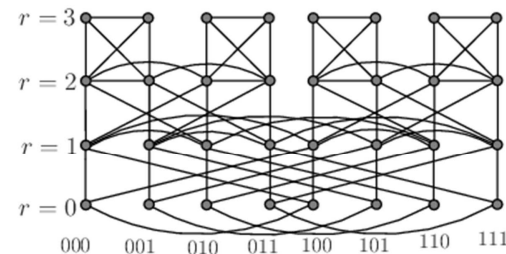

(2)
Figure 1. $A B_{2}$ and $A B_{3}$.

Let $n \geq 1$ be an integer. The vertices of the $n$-dimensional augmented butterfly network are the pairs $(r, x)$ where $r$ is a non-negative integer $0 \leq r \leq n$ called the level, and $x=\left(x_{1} x_{2} \cdots x_{n}\right)$ is a binary string of length $n$. In $A B_{n}$ the vertex $(r, x), 0 \leq r \leq n-1$, is adjacent to the vertices $(r+1, x)$, $\left(r+1, x_{1} x_{2} \cdots x_{r} \overline{x_{r+1}} x_{r+2} \cdots x_{n}\right),\left(r, x_{1} x_{2} \cdots x_{r-1} \overline{x_{r}} x_{r+1} \cdots x_{n}\right)$ and $\left(r, x_{1} x_{2} \cdots x_{r} \overline{x_{r+1}} x_{r+2} \cdots x_{n}\right)$. In particular, when $r=0$, the vertex $\left(0, x_{1} x_{2} x_{3} \cdots x_{n}\right)$ is adjacent to the vertices $\left(1, x_{1} x_{2} x_{3} \cdots x_{n}\right),\left(1, \overline{x_{1}} x_{2} x_{3} \cdots x_{n}\right)$ and $\left(0, \overline{x_{1}} x_{2} x_{3} \cdots x_{n}\right)$. Also when $r=n,\left(n, x_{1} x_{2} x_{3} \cdots x_{n}\right)$ is adjacent to the vertices $\left(n, x_{1} x_{2} x_{3} \cdots \overline{x_{n}}\right),\left(n-1, x_{1} x_{2} x_{3} \cdots x_{n}\right)$ and $\left(n-1, x_{1} x_{2} x_{3} \cdots \overline{x_{n}}\right)$.
Clearly $A B_{n}$ has $(n+1) 2^{n}$ vertices and $3 n \times 2^{n}$ edges [4]. $A B_{2}$ and $A B_{3}$ are shown in Figure 1(1) and Figure 1(2) respectively.

Let $r \in\{0,1,2, \ldots, n\}$, we define $V_{r}$ be the set of vertices of the form $(r, x)$, that is, the vertices in layer $r$. Let $i \in\{0,1\}$. Define $A B_{n}^{i}$ be the subgraph of $A B_{n}$ induced by vertices of the form $\left(r, i x^{\prime}\right)$ where $1 \leq r \leq n$ and $x^{\prime}$ is a binary string of length $n-1$. Note that $A B_{n}^{i}$ is isomorphic to $A B_{n-1}$. Thus for any $A B_{n}^{i}(i=0,1)$, let $j \in\{0,1\}$, the subgraph induced by the vertices of the form $\left(r, i j x^{\prime \prime}\right)$ where $2 \leq r \leq n$ and $x^{\prime \prime}$ is a binary string of length $n-2$, denoted by $A B_{n}^{i j}$, which is isomorphic to $A B_{n-2}$.

The edges between $(r, x) \quad$ and $\left(r, x_{1} x_{2} \cdots x_{r-1} \overline{x_{r}} x_{r+1} \cdots x_{n}\right), 0 \leq r \leq n$ and between $(r, x)$ and $\left(r, x_{1} x_{2} \cdots x_{r} \overline{x_{r+1}} \cdots x_{n}\right), 0 \leq r \leq n$ are called level edges. The edges between $(r, x)$ and $(r+1, x)$ are called straight edges while the edges between $(r, x)$ and $\left(r+1, x_{1} x_{2} \cdots x_{r} \overline{x_{r+1}} \cdots x_{n}\right)$ are called cross edges.

For complete graphs, Park and Ihm [7] derived the following results.

Lemma 2-1 [7]

For complete graph $K_{n}, \operatorname{smp}\left(K_{n}\right)=n-1$.

The conditional matching preclusion number of a graph $G$, denoted by $m p_{1}(G)$, with $n$ vertices is the minimum number of edges whose deletion results in graph without an isolated vertex and does not have a perfect matching if $n$ is even, or almost-perfect matching if $\mathrm{n}$ is odd. The conditional matching preclusion number for the Augmented Butterfly Network is given in a study [5].

Lemma 2-2

Let $G$ be the Augmented Butterfly Network $A B_{n}$, then $m p_{1}(G)=3$.

\section{Strong Matching Preclusion of Augmented Butterfly Network}

\section{Lemma 3-1}

For any two vertices of $A B_{2}$, say $u, v, A B_{2}-\{u, v\}$ has a perfect matching if and only if $\{u, v\} \neq\{(1,00),(1,11)\}$ and $\{(1,01),(1,10)\}$.

Proof. We can assume, without loss of generality, that $\{u, v\}=\{(1,00),(1,11)\} \quad$, then $A B_{2}-\{u, v\}$ has two components with odd vertices, clearly $A B_{2}-\{u, v\}$ has no perfect matching. It suffices to prove that $A B_{2}-\{u, v\}$ has a perfect matching if $\{u, v\} \neq\{(1,00),(1,11)\} \quad$ and $\{(1,01),(1,10)\}$. By the construction of $A B_{2}$, we can check the result easily, thus we omit the remainder proof here. 
From Lemma3-1, we know that for any vertex of $A B_{2}$, say $u$, and any vertex in layer 0 , say $\mathrm{v}$, there is a perfect matching of $A B_{2}-\{u, v\}$, say $M$, then $M \cup\{v\}$ is an almost perfect matching of $A B_{2}-u$. That means there exists an almost perfect matching of $A B_{2}-u$ such that any vertex in layer 0 can not matched.

Let $F=F_{V} \cup F_{E}, F_{V} \subseteq V\left(A B_{n}\right)$ and $F_{E} \subseteq E\left(A B_{n}\right)$.

Theorem 3-1

$\operatorname{smp}\left(A B_{2}\right)=2$.

Proof. From Lemma 3-1, $A B_{2}-\{(1,00),(1,11)\}$ or $A B_{2}-\{(1,01),(1,10)\}$ has no perfect matching, then $\operatorname{smp}\left(A B_{2}\right) \leq 2$. We need to show $\operatorname{smp}\left(A B_{2}\right) \geq 2$. It suffices to prove that for every $F$ and $|F|=1, A B_{2}-F$ has a perfect matching or an almost perfect matching. If $F=\{v\}$, as $A B_{2}$ has a perfect matching, $A B_{2}-F$ must have an almost perfect matching. If $F=\{e\}$, from Lemma 2-1, we know $A B_{2}-F$ must have a perfect matching. Thus $\operatorname{smp}\left(A B_{2}\right) \geq 2$, as desired.

Theorem 3-2

For $A B_{3}, \operatorname{smp}\left(A B_{3}\right)=3$.

Proof As $\delta\left(A B_{n}\right)=3, \operatorname{smp}\left(A B_{3}\right) \leq 3$. We need to show $\operatorname{smp}\left(A B_{3}\right) \geq 3$. It suffices prove that for each $F$ and $|F|=2$, $A B_{3}-F$ has a perfect matching or an almost perfect matching. If $F$ consists of two edges or one vertex and one edge, from Lemma 2-2, $A B_{3}-F$ has a perfect matching or an almost perfect matching. So we only need to consider that $\mathrm{F}$ consists of two vertices, say $F=\left\{v_{1}, v_{2}\right\}$, and complete the proof by distinguishing the following four cases.

Case 1. Both $v_{1}$ and $v_{2}$ are in the same $A B_{3}^{i}(i=0,1)$.

Without loss of generality, suppose $v_{1}, v_{2} \in V\left(A B_{3}^{0}\right)$. From Lemma 3-1, we know that if $\left\{v_{1}, v_{2}\right\} \neq\{(2,000),(2,011)\}$ and $\left\{v_{1}, v_{2}\right\} \neq\{(2,001),(2,010)\}$, then $A B_{3}^{0}-\left\{v_{1}, v_{2}\right\}$ has a perfect matching, say $M^{0}$, and $A B_{3}^{1}$ has a perfect matching, say $M^{1}$, then $M=M^{0} \cup M^{1} \cup\left(0,0 x_{2} x_{3}\right)\left(0,1 x_{2} x_{3}\right)\left(x_{2} x_{3}\right.$ is a binary string of length 2) is a perfect matching of $A B_{3}-F$. Now suppose $\left\{v_{1}, v_{2}\right\}=\{(2,000),(2,011)\}$, as well as the proof of $\left\{v_{1}, v_{2}\right\}=\{(2,001),(2,010)\} \quad$. From Lemma 3-1, $A B_{3}-\{(1,100),(1,111)\}$ has a perfect matching, say $M^{1}$. Let $M^{0}=(3,000)(3,001) \cup(3,010)(3,011)$, and $M^{2}$ be the set of all level edges in layer $0 . \quad$ Then $M=M^{0} \cup M^{1} \cup M^{2} \cup(2,011)(1,001) \cup(2,010)(1,010) \cup(1,000)$ $(1,100) \cup(1,011)(1,111)$ is a perfect matching of $A B_{3}-F$.

Case 2. $v_{1}$ and $v_{2}$ are in different $A B_{3}^{i}(i=0,1)$.

Without loss of generality, suppose $v_{1} \in V\left(A B_{3}^{0}\right)$ and $v_{2} \in V\left(A B_{3}^{1}\right)$. We can choose a vertex $u, u \in V_{1}$, $u \in V\left(A B_{3}^{0}\right), u \neq v_{1}$ such that $u$ is not adjacent to $v_{2}$, let $u^{\prime}$ be the neighbor of $u$ in $V_{1}$ and $u^{\prime} \in A B_{3}^{1}$. By Lemma 3-1, $A B_{3}^{0}-\left\{v_{1}, u\right\}$ has a perfect matching, say $M^{0}$, and $A B_{3}^{1}-\left\{v_{2}, u^{\prime}\right\}$ has a perfect matching, say $M^{1}$. All level edges of layer 0 induce a perfect matching of $V_{0}$, denoted by $M^{2}$. Then $M=M^{0} \cup M^{1} \cup M^{2} \cup\left(u u^{\prime}\right)$ is a perfect matching of $A B_{3}-\left\{v_{1}, v_{2}\right\}$.

Case 3. $v_{1} \in V\left(A B_{3}^{i}\right)(i=0,1)$ and $v_{2}$ is in layer 0 , that is $v_{2} \in V_{0}$.

Suppose $v_{1} \in V\left(A B_{3}^{0}\right), \quad v_{2}=\left(0, x_{1} x_{2} x_{3}\right), \quad\left(x_{1} x_{2} x_{3}\right)$ is a binary string of length 3 . Without loss of generality, assume $x_{1}=0$, and the proof can be obtained similarly if $x_{1}=1$. If $v_{1}=\left(1, x_{1} x_{2} x_{3}\right)$ or $\left(1, \overline{x_{1}} x_{2} x_{3}\right)$, this means $v_{1}$ is adjacent to $v_{2}$, say $v_{1}=\left(1, x_{1} x_{2} x_{3}\right)$. All straight edges between layer 2 and layer 3 induce a perfect matching of $V_{2} \cup V_{3}$, denoted by $M_{0}$. And $M_{1}=\left\{\left(0, x_{1} x_{2} x_{3}\right)\left(1, x_{1} x_{2} x_{3}\right)\right\} \backslash\left(v_{1} v_{2}\right)$, thus $M=M_{0} \cup M_{1}$ is a perfect matching of $A B_{3}-\left\{v_{1}, v_{2}\right\}$.

If $v_{1} \neq\left(1, x_{1} x_{2} x_{3}\right)$ and $\left(1, \overline{x_{1}} x_{2} x_{3}\right)$, this means $v_{1}$ is not adjacent to $v_{2}$. We know that $\left(0, \overline{x_{1}} x_{2} x_{3}\right)$ is adjacent to $v_{2}$ in layer 0 , and $\left(1, x_{1} x_{2} x_{3}\right)$ is adjacent to both $\left(0, \overline{x_{1}} x_{2} x_{3}\right)$ and $v_{2}$. From Theorem 3-1, $A B_{3}^{0}-\left\{v_{1},\left(1, x_{1} x_{2} x_{3}\right)\right\}$ has a perfect matching, say $M^{0}$, and $A B_{3}^{1}$ has a perfect matching, say $M^{1}$. Let $M^{2}$ be a perfect matching of $V_{0}-\left\{v_{2},\left(0, \overline{x_{1}} x_{2} x_{3}\right)\right\}$ induced by all level edges of layer 0 except the edge $\left(v_{2},\left(0, \overline{x_{1}} x_{2} x_{3}\right)\right)$, thus $M=M^{0} \cup M^{1} \cup M^{2} \cup\left(\left(0, \overline{x_{1}} x_{2} x_{3}\right)\left(1, x_{1} x_{2} x_{3}\right)\right)$ is a perfect matching of $A B_{3}-\left\{v_{1}, v_{2}\right\}$.

Case 4. Both $v_{1}$ and $v_{2}$ are in layer 0 .

Let $v_{1}=\left(0, x_{1} x_{2} x_{3}\right), \quad v_{2}=\left(0, y_{1} y_{2} y_{3}\right), \quad\left(x_{1} x_{2} x_{3}\right) \quad$ and $\left(y_{1} y_{2} y_{3}\right)$ is a binary string of length 3 . If $\left(y_{1} y_{2} y_{3}\right)=\left(\overline{x_{1}} x_{2} x_{3}\right)$, implying that $v_{1}$ is adjacent to $v_{2}$. As $A B_{3}^{0}$ and $A B_{3}^{1}$ have a perfect matching respectively, say $M^{0}$ and $M^{1}$. The level edges of layer 0 except $\left(v_{1} v_{2}\right)$ induce a perfect of $V_{0}-\left\{v_{1}, v_{2}\right\}$, denoted by $M^{2}$. Then $M=M^{0} \cup M^{1} \cup M^{2} \cup\left(v_{1} v_{2}\right)$ is a perfect matching of $A B_{3}-\left\{v_{1}, v_{2}\right\}$.

If $\left(y_{1} y_{2} y_{3}\right) \neq\left(\overline{x_{1}} x_{2} x_{3}\right)$, that is $v_{1}$ isn't adjacent to $v_{2}$. Let $u_{1}$ and $u_{2}$ be the neighbors of $v_{1}$ and $v_{2}$ in layer 0 respectively, and $u_{1}^{1}, u_{2}^{1}$ be the neighbors of $u_{1}$ and $u_{2}$ of $A B_{3}^{1}$ in layer 1 respectively. And from Theorem 3-1, $A B_{3}^{1}-\left\{u_{1}^{1}, u_{2}^{1}\right\}$ has a perfect matching, say $M^{1}, A B_{3}^{0}$ has a perfect matching, say $M^{0}$. The level edges of layer 0 except $\left\{\left(v_{1} u_{1}\right),\left(v_{2} u_{2}\right)\right\}$ induce a perfect matching of 
$V_{0}-\left\{v_{1}, v_{2}, u_{1}, u_{2}\right\}$, denoted by $M^{2}$.

Thus $M=M^{0} \cup M^{1} \cup M^{2} \cup\left\{\left(u_{1} u_{1}^{1}\right),\left(u_{2} u_{2}^{1}\right)\right\}$ is a perfect matching of $A B_{3}-\left\{v_{1}, v_{2}\right\}$, completing the proof.

Theorem 3-3

If $n \geq 4, \operatorname{smp}\left(A B_{n}\right)=3$.

Proof. We complete the proof by induction on $n$. The result is true for $n=3$ by Theorem 3-2. Assume that the result is true for $A B_{n-1}$. As $\delta\left(A B_{n}\right)=3, \operatorname{smp}\left(A B_{n}\right) \leq 3$. We need to show $\operatorname{smp}\left(A B_{n}\right) \geq 3$. It suffices to prove that for each $F$ and $|F|=2, A B_{n}-F$ has a perfect matching or an almost perfect matching. If $F$ contains at least one edge, that is $F$ contains two edges or one edge and one vertex, then by Lemma 2-2, $A B_{n}-F$ has a perfect matching or an almost perfect matching. Now we only need to consider that $F$ consists of two vertices, say $F=\left\{v_{1}, v_{2}\right\}$, and complete the proof by the following cases.

Case 1. $v_{1}$ and $v_{2}$ are in different $A B_{n}^{i}(i=0,1)$.

Without loss of generality, let $v_{1} \in V\left(A B_{n}^{0}\right)$ and $v_{2} \in V\left(A B_{n}^{1}\right)$. We can choose a vertex of $V_{1}$, say $u$, $u \in V\left(A B_{n}^{0}\right), u \neq v_{1}$ and $u$ is not adjacent to $v_{2}$, let $\mathrm{u}^{\prime}$ be the vertex adjacent to $u$ in layer 1 . By induction hypothesis, $A B_{n-1}^{0}-\left\{v_{1}, u\right\}$ has a perfect matching, say $M^{0}$, and $A B_{n-1}^{1}-\left\{v_{2}, u^{\prime}\right\}$ has a perfect matching, say $M^{1}$. Denote the perfect matching of $V_{0}$ induced by all the level edges in layer 0 by $M^{2}$. Then $M=M^{0} \cup M^{1} \cup M^{1} \cup M^{2} \cup\left(u u^{\prime}\right)$ is a perfect matching of $A B_{n}-\left\{v_{1}, v_{2}\right\}$.

Case 2. $v_{1} \in V\left(A B_{n}^{i}\right)(i=0,1)$ and $v_{2}$ is in layer 0 .

Suppose $v_{1} \in V\left(A B_{n}^{0}\right), \quad v_{2}=\left(0, x_{1} x_{2} \cdots x_{n}\right)$. If $v_{1}$ is adjacent to $v_{2}$. Denote a perfect matching of $A B_{n}^{i j}$ by $M^{i j}(i, j=0,1)$, and let $M^{2}$ be the perfect matching of $V_{0} \cup V_{1}-\left\{v_{1}, v_{2}\right\}$ induced by the straight edges between layer 0 and layer 1 except the edge $\left(v_{1} v_{2}\right)$. Then $M=M^{00} \cup M^{01} \cup M^{10} \cup M^{11} \cup M^{2}$ is a perfect matching of $A B_{n}-\left\{v_{1}, v_{2}\right\}$.

If $v_{1}$ is not adjacent to $v_{2}$. There is a vertex in layer 0 which is adjacent to $v_{2}$, say $v_{2}^{\prime}$, and the vertex of $A B_{n}^{0}$ in layer 1 which is adjacent to $v_{2}$, denoted by $v_{2}^{\prime \prime}$. By induction hypothesis, $A B_{n}^{0}-\left\{v_{1}, v_{2}^{\prime \prime}\right\}$ has a perfect matching, say $M^{0}$. Choose a perfect matching of $A B_{n}^{1}$, say $M^{1}$. Let $M^{2}$ be the perfect matching of $V_{0}-\left\{v_{2}, v_{2}^{\prime}\right\}$ induced by the level edges in layer 0 except $\left(v_{2} v_{2}^{\prime}\right)$. Then $M=M^{0} \cup M^{1} \cup M^{2} \cup\left(v_{2}^{\prime} v_{2}^{\prime \prime}\right)$ is a perfect matching of $A B_{n}-\left\{v_{1}, v_{2}\right\}$.

Case 3. Both $v_{1}$ and $v_{2}$ are in layer 0 .

Suppose $v_{1}=\left(0, x_{1} x_{2} \cdots x_{n}\right)$ and $v_{2}=\left(0, y_{1} y_{2} \cdots y_{n}\right)$. If $\left(y_{1} y_{2} \cdots y_{n}\right)=\left(\overline{x_{1}} x_{2} \cdots x_{n}\right)$, that means $v_{1}$ is adjacent to $v_{2}$, then $A B_{n}^{i}(i=0,1)$ has a perfect matching, say $M^{i}$. All level edges in layer 0 except $\left(v_{1} v_{2}\right)$ induce a perfect matching of $V_{0}-\left\{v_{1}, v_{2}\right\}$, denoted by $M^{2}$. Thus $M=M^{0} \cup M^{1} \cup M^{2}$ is a perfect matching of $A B_{n}-\left\{v_{1}, v_{2}\right\}$.

If $\left(y_{1} y_{2} \cdots y_{n}\right) \neq\left(\overline{x_{1}} x_{2} \cdots x_{n}\right)$, that means $v_{1}$ isn't adjacent to $v_{2}$. Let $v_{1}^{\prime}=\left(0, \overline{x_{1}} x_{2} \cdots x_{n}\right)$ and $v_{2}^{\prime}=\left(0, \overline{y_{1}} y_{2} \cdots y_{n}\right)$, and $v_{i}^{\prime \prime}(i=1,2)$ is the vertex of $A B_{n}^{0}$ in layer 1 which is adjacent to $v_{i}^{\prime}(i=1,2)$. From induction hypothesis, $A B_{n}^{0}-\left\{v_{1}^{\prime \prime}, v_{2}^{\prime \prime}\right\}$ has a perfect matching, say $M^{0}$, and $A B_{n}^{1}$ has a perfect matching, say $M^{1}$. Let $M^{2}$ be the perfect matching of $V_{0}-\left\{v_{1}, v_{2}, v_{1}^{\prime}, v_{2}^{\prime}\right\}$ induced by the level edges of layer 0 except $\quad\left(v_{1} v_{1}^{\prime}\right)$ and $\left(v_{2} v_{2}^{\prime}\right)$. Thus $M=M^{0} \cup M^{1} \cup M^{2} \cup\left(v_{1}^{\prime} v_{1}^{\prime \prime}\right) \cup\left(v_{2}^{\prime} v_{2}^{\prime \prime}\right)$ is a perfect matching of $A B_{n}-\left\{v_{1}, v_{2}\right\}$, completing the proof.

\section{Conclusions}

The problem of investigating the strong matching preclusion of interconnection networks is very difficult as strong matching preclusion that additionally permits more destructive vertex faults in a graph is a more extensive form of the original matching preclusion that assumes only edge faults. Augmented Butterfly networks is an important network, in this paper, the strong matching preclusion number for n-dimension Augmented Butterfly networks is obtained by induction hypothesis. Furthermore, determining the strong matching preclusion number for other interconnection networks are under study.

\section{Acknowledgements}

Supported by the Ministry of Education Chunhui Plan (No.2107z046), National Science Foundation of China.

(Nos. 11601254, 11661068, 61763041, and 11551001) and the Science Found of Qinghai Province (Nos. 2019-ZJ-921).

\section{References}

[1] P. Bonnevilie, E. Cheng, J. Renzi, strong matching preclusion for the alternating group graphs and split-stars, $J$. Interconnection. Netw., 2011, Vol. 12, No. 4, 277-298.

[2] R. C. Brigham, F. Harary, E. C. Violin, J. Yellen, perfect matching preclusion, Congr. Numer 2005, 185-192.

[3] J. Bondy, U. Murty, Graph Theory, GTM244, Springer, 2008.

[4] P. Manuel, I. Rajasingh, B. Rajan and Prabha, Augmented Butterfly Network, J. Combin. Inform. Syst. Sci. 2008, 33, 27-35.

[5] M. J. Raja, D. A. Xavier. Conditional Matching Preclusion Number for Butterfly Derived Networks. Inter. J. Pure Appl. Math. 2016, 7, 17-25. 
[6] J.-H. Park, Matching preclusion problem in restricted HL-graphs and recursive circulate $g(2 \mathrm{~m}, 4)$, Journal of KIISE 2008, 35, 60-65.

[7] J.-H. Park, I. Ihm, Strong matching preclusion, Theor. Comput. Sci. 2011, 412, 6409-6419.

[8] J.-H. Park, I. Ihm, Strong matching preclusion under the conditional fault model, Discrete Appl. Math 2013, 161, 1093-1105.

[9] E. Cheng, J. Kelm, J. Renzi, Strong matching preclusion of (n, k)-star graphs, Theory. Comput. Sci. 2016, 615, 91--101.

[10] E. Cheng, D. Lu, B. Xu, Strong matching preclusion of pancake graphs, J. Interconnection. Netw 2013, 14 (2), 1350007.

[11] E. Cheng, S. Shah, V. Shah, D. E. Steffy, Strong matching preclusion for augmented cubes, Theor. Comput. Sci. 2013, 491, 71-77.
[12] S. Wang, K. Feng, G. Zhang, Strong matching preclusion for k-ary n-cubes, Discrete Appl. Math 2013, 161, 3054-3062.

[13] X. M. Hu, Y. Z. Tian, X. D. Liang, J. X. Meng, Strong matching preclusion for n-dimensional torus networks, Theor. Comput. Sci 2016, 635, 64-73.

[14] X. M. Hu, Y. Z. Tian, X. D. Liang, J. X. Meng, Strong matching preclusion for k-composition networks, Theor. Comput. Sci 2018, 711, 36-43.

[15] Y. Mao, Z. Wang, E. Cheng, C. Melekian, Strong matching preclusionnumber of graphs, Theor. Comput. Sci. 2018, 713, $11-20$.

[16] Z. Wang, Y. Mao, E. Cheng, J. Y. Zou, Matching preclusion number of graphs, Theor. Comput. Sci 2019, 759, 61-71. 